\title{
La concordancia de la frase nominal por los sino hablantes aprendices de español como segunda lengua*.
}

\author{
Noun phrase concordance in learners of Spanish \\ as a second language
}

\author{
Daniela Gutiérrez Barraza \\ Estudiante de Posgrado en Humanidades \\ Universidad de Sonora \\ Hermosillo, Sonora, México \\ e-mail: danyg60@hotmail.com
}

Received: 31-Oct-2012 / Accepted: 4-June-2013

\section{Resumen}

En los últimos años se ha elevado el número de personas que quieren aprender español como segunda lengua/lengua extranjera. Por ello, es importante estudiar las dificultades que tienen que afrontar los estudiantes no nativos de español al momento de involucrarse en dicho proceso. Específicamente, los estudiantes hablantes nativos de chino mandarín enfrentan problemas particulares, puesto que tipológicamente es una lengua distinta al español, como el de la concordancia en las frases nominales. En el presente artículo presento datos donde no se establecen las relaciones de concordancia entre los elementos que conforman la frase nominal. Los datos recogidos, de dos estudiantes sino hablantes de los cursos de español del nivel intermedio en el Departamento de Lenguas Extranjeras de la Universidad de Sonora, consisten en textos que se obtuvieron a partir de ejercicios de clase, libro o elaborados por el maestro responsable del grupo, así como de la observación de imágenes y videos. Se encontró que la estructura de la frase nominal de los sino hablantes no es diferente del patrón esperado en español, sin embargo en ocasiones no establecen las relaciones de concordancia en género y número entre el núcleo nominal y el artículo, o el adjetivo.

Palabras claves: frase nominal, español como segunda lengua, concordancia nominal, modalidad escrita.

\section{Abstract}

In recent years the number of people who want to learn Spanish as a second / foreign language has increased. It is therefore important to study the difficulties that non-native students of Spanish have to face at the time they are involved in this process. Specifically, the native speakers students of Mandarin Chinese face particular problems because typologically it is a language different to Spanish, such as of the concordance in noun phrases. In this article I present data where there are not established relations of concordance between the elements that compose the noun phrase. Data collected from two students "but speaking" of the Spanish courses at the intermediate level in the Department of Foreign Languages at the Universidad de Sonora, consist of texts obtained from class exercises, book or prepared by the teacher responsible for the group, as well as the observation of images and videos. It was found that the structure of the nominal phrase of the "but speakers" is not different from the expected pattern in Spanish, though sometimes the relations of concordance are not set on gender and number between the nominal core and the article, or the adjective.

Keywords: noun phrase, Spanish as a second language, nominal concordance, written modality.

\section{Résumé}

Au cours des dernières années, le nombre de personnes qui souhaitent apprendre l'Espagnol comme seconde langue/langue étrangère s'est élevé. Aussi, il est important d'étudier les difficultés que les étudiants peuvent avoir au cours de leurs études. Particulièrement, les

* Este artículo refleja los resultados de la investigación titulada “Español. Estudios disciplinares, interdisciplinares y aplicaciones”. Llevada a cabo en la Universidad de Sonora, Hermosillo, Sonora, México. Durante los mese de enero 2011 a noviembre 2012. 
étudiants dont leur langue maternelle est le Chinois mandarin font face à des problèmes spécifiques, puisque la langue Chinoise est une langue typologiquement différente de l'Espagnol, tel le cas de la concordance dans des phrases nominales. Dans cet article, je présente des données où les relations de concordance entre les éléments qui composent la phrase nominale ne sont pas établies. Les données recueillies, provenant de deux étudiants sinophones des cours d'Espagnol au niveau intermédiaire du Département de Langues Étrangères de l'Université de Sonora, consistent en des textes obtenus à partir d'exercices faits dans la classe, dans le livre ou préparés par l'enseignant responsable du groupe, ainsi que de l'observation d'images et de vidéos. On a trouvé que la structure de la phrase nominale des sinophones n'est pas différente du modèle attendu en Espagnol, mais parfois ils ne font pas les concordances en genre et nombre entre le noyau nominal et l'article ou l'adjectif.

Mots clés : phrase nominale, l'Espagnol comme seconde langue, concordance nominale, modalité écrite.

\section{Resumo}

Nos últimos anos tem se elevado o número de pessoas que querem aprender espanhol como segunda língua/língua estrangeira. Por isso, é importante estudar as dificuldades que os estudantes não nativos de espanhol têm que afrontar no momento de envolver-se em dito processo. Especificamente, os estudantes falantes nativos de chinês mandarim enfrentam problemas particulares, posto que topologicamente, é uma língua diferente ao espanhol, como o da concordância nas frases nominais. No presente artigo apresento dados onde não se estabelecem as relações de concordância entre os elementos que conformam a frase nominal. Os dados recolhidos, de dois estudantes senão falantes dos cursos de espanhol do nível intermédio no Departamento de Línguas Estrangeiras da Universidade de Sonora consistem em textos que se obtiveram a partir de exercícios de aula, livro, ou elaborados pelo professor responsável do grupo, assim como da observação de imagens e vídeos. Encontrou-se que a estrutura da frase nominal dos senão falantes não é diferente do padrão esperado em espanhol, entretanto, em ocasiões não estabelecem as relações de concordância em gênero e número entre o núcleo nominal e o artigo, ou o adjetivo.

Palavras chaves: frase nominal, espanhol como segunda língua, concordância nominal, modalidade escrita.

\section{Introducción}

Actualmente el español es una de las lenguas que ocupa un lugar relevante dentro del ámbito del aprendizaje de segundas lenguas (L2) y lenguas extranjeras (LE), puesto que se ha incrementado el número de personas de distintas lenguas que han optado por estudiar el español como L2 o LE. Específicamente, en China se ha elevado la demanda de la enseñanza del español debido a factores del ámbito profesional, así como por motivos económicos, políticos y culturales.

Debido al interés por el aprendizaje del español por los sino hablantes y por las dificultades que enfrentan como estudiantes es que nace este trabajo. El español y el chino mandarín son dos lenguas que pertenecen a tipologías distintas por lo que habrá dificultades al momento de estructurar una palabra, oración o frase. En el presente estudio analizo la estructura de las frases nominales producidas por los estudiantes sino hablantes, que cursan el nivel intermedio de español del Departamento de Lenguas Extranjeras de la Universidad de Sonora. El objetivo es identificar los problemas de concordancia en género y número entre los elementos que constituyen estas frases nominales, con el fin de comprender cómo las diferencias que hay entre ambas lenguas, en cuanto a las construcciones nominales, afectan en la producción de los chino hablantes; también tiene como objetivo una orientación didáctica a hablantes de otras lenguas tipológicamente diferentes al español, brindando un material didáctico al maestro que apoye al ámbito de la enseñanza del español como segunda lengua/lengua extranjera.

Existen muchos estudios dentro del área de español como L2 a partir de distintos ámbitos, en especial en el campo de la enseñanza-aprendizaje (Charters, 2005; Cadierno, 2010; Murillo, 2004; Madrigal, 2008; Livinstone, 2009), y en la lingüística (Arcos, 2009; Bartolomé, 2008; Suárez, 2008; Liao y Fukuya, 2004); pero son escasos aquellos que estudian la frase nominal en español como L2.

En términos tipológicos, el chino mandarín es una lengua aislante, es decir, aquella en la que a cada palabra le corresponde un morfema, como en (1). Por otro lado, el español es una lengua fusional ya 
que los morfemas marcan varios significados, véase (2) en donde el verbo caminó indica tercera persona, singular, tiempo pasado, modo indicativo y aspecto perfectivo (Payne, 1997; Comrie, 1989).

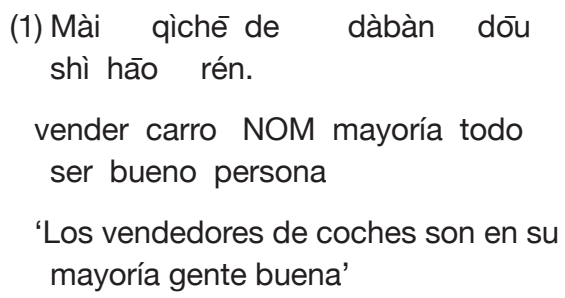

(2) María caminó en el parque durante una hora.

En español se encuentran los morfemas gramaticales (Alcina y Blecua, 2001; González y Herrero, 1997; Di Tullio, 2010; RAE, 2010), que son los "morfemas que añaden otros significados a la palabra" (González y Herrero, 1997:23); significados como pluralidad, tiempo, y más. Dentro de estos morfemas se hallan los flexivos, morfemas que involucran cambios gramaticales y establecen las relaciones sintácticas entre las palabras como la concordancia. Dentro de la frase nominal, la concordancia es la relación interna que hay entre el nombre y los elementos que lo acompañan en dicha frase, por medio de los morfemas de género y número (Alcina y Blecua, 2001:512; RAE, 2010:6). Gutiérrez (2004:115) dice que la concordancia es una de las dificultades a la que se tienen que enfrentar los estudiantes extranjeros que están aprendiendo el español como segunda lengua/lengua extranjera, más aún si estos morfemas nominales son muy distintos a los de su lengua materna.

Por otro lado, la morfología flexiva del español se comporta de manera distinta en comparación con el chino mandarín. Norman (1988) presenta un extenso estudio de esta lengua, mencionando que en el chino mandarín no hay presencia de morfemas que marquen relaciones gramaticales como es el caso del español, pero existen otros elementos para establecer estas relaciones. Dicho autor dice que "el orden de palabra, las partículas y las preposiciones son los encargados de mostrar cómo los elementos de una oración están relacionados unos con otros". Así, en el chino mandarín, no hay marcación de número ni de género en los nombres. Por ejemplo, Li y Thompson (1989) señalan que el nombre $s h \square$, hace referencia tanto a 'libro' como 'libros'. Un caso especial son los pronombres personales y posesivos, ya que llevan marcación de plural por medio del sufijo -men, como en $w \square$ men 'nosotros' y zánmende 'nuestro'; esta marcación también se utiliza en nombres que hacen referencia a seres humanos, como háizimen 'niños', donde háizi significa 'niño' que unido a -men ('un cierto grupo de') da como resultado 'niños', aunque con algunas restricciones (Po-Ching y Rimmington, 2004). Tampoco se expresa morfológicamente la categoría de género, pero este aspecto se maneja más bien en términos de referencia; ni en $y \square$ ge rén 'una persona' o $y \square g e y \square s h \square n g$ 'un doctor' no hay marca morfológica de género para los nombres; igualmente hay ausencia del artículo.

En cuanto a la frase nominal, en español es una construcción que no contiene verbo y está constituida por un núcleo, representado por un nombre o por otro elemento que funcione como tal. Se añaden otros elementos que pueden ser opcionales, es decir, pueden o no aparecer, dependiendo de la clase de palabra que se trate: 1 ) antes del nombre aparece: artículo, cuantificador, demostrativo, numeral, adjetivo calificativo, y 2) después del nombre: otro nombre, adjetivo, una frase prepositiva, una oración de relativo; dependiendo del tipo de adjetivo, puede aparecer antes o después del nombre; aquellos que ocupan ambas posiciones, denotaran significados diferentes (Alcina y Blecua, 2001; Alarcos, 1994). Ejemplo:

(3) Hace dos días, María fue a ver un doctor que su amiga Ana le había recomendado porque tenía mucho dolor en las rodillas. Después de verlo, ella se fue a su casa a descansar.

En chino mandarín, Li y Thompson (1989:104) declaran la estructura de una frase nominal consiste 
en al menos un pronombre, como $w \square$ 'yo', o un nombre, como $m \square$ 'caballo', y dicho nombre puede ser tanto un nombre simple como un nombre compuesto, tal como sh $\square b \square o$ 'bolsa de libros', o diànd $\square n g$ 'luz eléctrica'. Además, puede haber otros elementos los cuales deben de estar antes del nombre. Tales elementos son 1) frases clasificadoras y frases de medida, 2) frases asociativas, y 3) frases modificadoras. Estos tres elementos pueden ser opcionales excepto por el nombre, pero si aparecen todos, Li y Thompson (1989:124) establecen el siguiente esquema mostrando el orden de dichos elementos:

(4) Estructura de la frase nominal en chino mandarín:

a. frase asociativa + frase clasificadora / medida + clausula relativa + adjetivo + nombre.

b. frase asociativa + clausula relativa + frase clasificadora / medida + adjetivo + nombre.

c. frase clasificadora + frase asociativa + nombre

Donde una frase clasificadora es una frase compuesta por un demostrativo, como zhèi 'este' o nèi 'ese'; y/o por un número, tal como yí 'uno', bàn 'mitad', o shí 'diez'; o por un cuantificador, como zh】ng 'todo', $j \square$ 'cuantos' o 'unos pocos'; más el clasificador. Dicho clasificador (CL) es un morfema independiente que va antes del nombre (Li y Thompson 1989:104-105). Véase el siguiente ejemplo donde el clasificador ge está antes del nombre 'persona', y lo acompaña un morfema de número, $s \square n$ 'tres', siendo la frase clasificadora $s \square n-g e$ :

(5) $[s \cap n-g e]$ rén tres-CL persona

'Tres personas'.

El uso de un clasificador estará determinado por el nombre que acompaña, es decir, que el criterio de uso está regido por medio de las propiedades naturales del nombre, por ejemplo, el clasificador $b \square$ se usa para objetos que pueden ser recogidos por una mano, o tái para equipo eléctrico; pero no es determinado solamente por factores semánticos o pragmáticos, ya que por ejemplo un teléfono puede ser agarrado por una mano pero el clasificador correcto que lo acompaña es tài (Charters, 2005:202).

Las frases de medida, como su nombre lo indica, son morfemas independientes que expresan longitud, peso, área o volumen, además de indicar agregados o contenedores (Li y Thompson, 1989:105):

(6) a. shí bàng

diez libra

'Diez libras'

b. wu - shí píng yóu

cinco - díez botella aceite

'Cincuenta botellas de aceite'.

En (6a) la frase de medida viene formada por un número shí 'diez' seguido de un morfema independiente de medida bàng 'libra' pero funcionando como nombre, no como clasificador. $Y$ en (6b) se tiene una frase de medida que indica contenedor, compuesta por dos números wu-shí 'cinco-diez' y un morfema independiente de contenedor de medida píng 'botella', seguida del nombre yóu 'aceite'.

Una frase asociativa indica tanto asociación como posesión, y está estructurada por dos frases nominales unidas por medio de la partícula -de, cuyo significado total es la suma de los significados de las dos frases. La primera frase nominal se considera la frase asociativa, y la segunda, es el núcleo nominal que será modificado (Li y Thompson (1989:113).

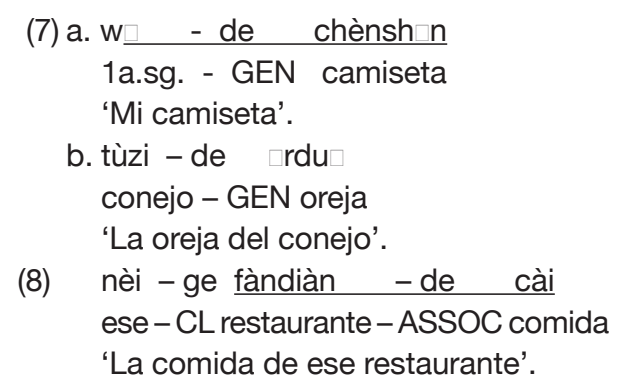

Por último, las frases modificadoras tienen la función de ser una cláusula relativa o un atributo 
adjetival. Cuando aparece una cláusula relativa significa que hay una cláusula nominalizada que modifica al nombre, y cuando se tiene un adjetivo atributivo es cuando hay un adjetivo que modifica a un nombre en una frase nominal (Li y Thompson, 1989:116). Véase lo siguiente:
(9) at shi yi -ge $\underline{\text { ho }}$ rén 3a.sg. ser uno - CL buena persona 'Él /ella es una buena persona'.

(10)
a. hóng de hu rojo NOM flor
' Una flor que es roja'.
b. hóng hu
rojo flor
'Una flor roja'.

En (9) se tiene un adjetivo atributivo, ya que el adjetivo $h \square$ o 'buena' modifican al nombre que le sigue, rén 'persona'; el adjetivo atributivo es la frase modificadora. Mientras que en (10a) el adjetivo hóng 'rojo' modifica al nombre $h u \square$ 'flor', pero en (10b) el adjetivo aparece dentro de una cláusula relativa pues viene acompañado de la partícula de. La diferencia es que el adjetivo en una clausula relativa sirve para especificar cómo es el núcleo nominal, es decir, nos hace saber cómo es el referente, pero al no aparecer en una cláusula relativa lo que hace el adjetivo es marcar una categoría de entidades.

\section{Metodología}

Los datos para este trabajo son producciones escritas, obtenidas de la manera lo más espontáneamente posible, de dos estudiantes sino hablantes de la clase de español de nivel intermedio del Departamento de Lenguas Extranjeras de la Universidad de Sonora en Hermosillo, Sonora, México. Dichos cursos van dirigidos no solo a estudiantes universitarios de intercambio sino a todo aquel que quiera aprender el español con hablantes nativos de esta ciudad. El objetivo principal es que el estudiante desarrolle las habilidades lingüísticas básicas para poderse comunicar: hablar, leer, escuchar y escribir; $y$ además de conocer no solo la cultura mexicana sino también la cultura regional sonorense.

El corpus está compuesto por 40 producciones escritas cuya extensión es variada, puesto que se componen desde un conjunto pequeño de oraciones aisladas hasta textos que van de dos a cuatro párrafos. Estos textos fueron obtenidos a partir de los ejercicios que les daba la maestra a cargo del grupo en el aula. Algunos eran ejercicios del libro que utilizaban en clase, otros eran liderados por la maestra, y otros elaborados por el investigador. Dichos ejercicios eran en base al tema que se estaba viendo en clase, y otros eran como una actividad libre, donde escribían sobre algún tema de interés personal. Después, la obtención de datos se llevó a cabo de manera individual, dentro y fuera del aula. Básicamente se siguió la misma dinámica, esto es, se presentaba un tema, se discutía, y se continuaba con una serie de ejercicios. Los escritos que se produjeron exponían opiniones sobre lo que se había dialogado, otros textos surgieron a partir de preguntas de aspectos más personales, sobre la cultura, gustos, si tuviera dinero ċqué haría con él?, incluso se utilizaron cortometrajes para que después comentara lo que había tratado la historia.

\section{Resultados}

Como se pudo observar previamente, la estructura de las frases nominales del chino mandarín es diferente a las del español, pues el mandarín no cuenta con los mismos elementos que utiliza el español tales como los artículos, marcas morfológicas para indicar género y número entre los elementos, así como de marcación de tiempo, aspectos esenciales para estructurar el discurso en el español. El chino mandarín se ayuda del contexto o de la situación para obtener el significado preciso, además se basa en el referente para poder emplear algunos elementos que constituyen una frase nominal, como los clasificadores, de manera que toma en consideración las características de la entidad de la que se habla.

Lo que encontré es que se presentaron frases nominales en donde no se daba las relaciones de 
concordancia de género y número, entre el núcleo nominal y artículos, y entre el núcleo nominal y sus modificadores. En total se presentaron 523 frases nominales, cuya estructura no difiere mucho al del patrón típico del español. Las que más se hicieron presentes fue la del artículo más nombre. Sin embargo, como se puede ver en el cuadro 1 , algunas estructuras nominales presentaron problemas:

Con respecto a la ausencia de género, se presentó entre el artículo, el demostrativo, cuantificador, y el

\begin{tabular}{|l|c|c|c|}
\hline Estructura de la frase nominal & & \multicolumn{2}{|c|}{ Ausencia de concordancia } \\
\hline ART + N & $14 / 199$ & 10 & 5 \\
\hline $\mathrm{N}$ & $1 / 102$ & & \\
\hline $\mathrm{N}+$ ADJ.CAL & $3 / 19$ & 3 & \\
\hline ART + N + ADJ.CAL & $7 / 18$ & 6 & 1 \\
\hline ADJ.CAL + N & $1 / 5$ & & 1 \\
\hline ART + N + N + ADJ.CAL & $1 / 3$ & 1 & \\
\hline Total & $27 / 346$ & 20 & 7 \\
\hline
\end{tabular}

Cuadro 1. Frases nominales con ausencia de concordancia en género y número.

núcleo nominal, así como con algunos adjetivos que le seguían.

(11) a. (...) no me gusta la clima de este ciudad" (A1T05)

b. "Mira, una corba está comiendo un liebre" (L2T18)

c. "Este semana santa" (...) (L1T06)

d. "Tenía muchas problemas pero (...)" (L1T08)

En (11a y b), se tiene la falta de concordancia entre el artículo definido (11a) y el artículo indefinido (11b) en relación con el referente "clima" y "liebre" respectivamente. Ambos nominales son excepciones de la regla general de marcación de género, ya que "clima" aunque termina con el sufijo $-a$, hace referencia al género masculino, y "liebre", expresa género femenino aunque no tenga el morfema -a. Por ello, el estudiante posiblemente se basó en esta terminación morfológica para establecer la concordancia en español.

En (11a) y (11c) se halla el demostrativo "este", elemento que en los datos aparece siempre de la misma forma con cualquier elemento nominal. "Este" hace referencia a un objeto cercano masculino, pero en ambos casos aparece con un nominal femenino, "ciudad" y "semana". En (11d) se presenta el cuantificador "muchas", elemento que también aparece en el chino mandarín, pero también hay ausencia de concordancia pues lo acompaña el nombre "problemas", que es masculino. En este caso, considero también que se basó en la regla general de concordancia.

En (12), se tienen ejemplos cuyos referentes nominales expresan género femenino, y si bien concuerdan con el artículo que le anteceden, no lo hacen con el adjetivo que los acompañan. Todos estos adjetivos aparecen en masculino, para los ejemplos (12b) y (12c), se repite la misma situación en ambos estudiantes. En (12d) hay presencia de preposición para relacionar el atributo con el nombre, más no es necesario.

(12) a. "La menta es una planta bajo y puqueño" (L1T02)

b. "Y hacia una fiesta modeno por Internt (...)" (L1T06)

c. "Ahoro, Beijing es una ciudad moderno y urbanización" (A1T01)

d. "(...) la persona de minusválido" (L2T20)

Como se puede observar, no hay dificultades en cuanto al orden de los elementos dentro de las 
frases nominales. El problema radica en que las relaciones de concordancia entre el núcleo nominal y algún otro elemento que lo acompaña no están bien establecidas.

Como lo muestra el cuadro 1 , se encontró con menor frecuencia la ausencia de concordancia de número. Algunos de los casos, son los de (13) y (14).

(13)a. "Entonce ellos caminan con sus pie" (L2T29)

b. "A mí me encantan las película de KongFu" (L1LT05)

c. "Hace un años yo llegué aquí" (L1T08)

En (13a) tenemos el pronombre posesivo de tercera persona plural "sus" que no establece relación de número con el nombre al que hace referencia, pues está en singular. En el chino mandarín, como se vio, no hay marcación de pluralidad en los nombres, más sin embargo, Li y Thompson (1989: 12), dicen que el único lugar donde el chino mandarín debe de marcar pluralidad es en los pronombres, -men. En (13b) y (13c) tiene que ver más con el referente, "película" y "años". En (13b) "película de KongFu" denota una categoría o un tipo de película, por lo que no es necesario marcar plural, pues el chino mandarín no lo hace (en el caso de los clasificadores), y por ello se apoya con el artículo "las" para marcar dicha pluralidad, que son todas las películas de ese tipo las que le gusta. Y con el nombre "años", este elemento nominal denota ya en sí un período largo de tiempo, por ello ya automáticamente por la referencia lo expresa como plural. En realidad, tenía un año de haber llegado a la ciudad de Hermosillo, Sonora.

Ahora bien, en (14a) se tiene un adjetivo que le precede al nombre, cuya marcación viene marcada en plural, pero una vez establecido que se trata de más de una entidad, el adjetivo, como en el chino mandarín, no necesita marcar número de plural. En el caso de (14b), se tiene un nombre de masa 'tiempos', pero acompañado por un cuantificador 'muchos'. En chino, se utilizan los cuantificadores para poder indicar pluralización, y como ya en sí el nominal 'tiempo' indica 'una cantidad de' (minutos, horas, días, etc.) entonces también lo expresa en el cuantificador, marcándolo también con el plural.

(14)a. "(...) pero estaban en diferente países" (L1T06)

b. "Tenía muchos tiempos para todos"

(A1T03)

Con respecto al artículo, como se pudo ver arriba, también hubo falta de concordancia con el nominal que le seguía; hay que recordar que el chino mandarín no tiene este elemento. En los siguientes ejemplos al presentarse nominales como 'lugar' e 'isla' se presentaron problemas en cuanto al género del artículo que los acompañaban:

(15)a. "porque ahora toda gente trabaja muy duro no hay una lugar más tranquila"

b. "Las islas son muy bunitas están en el océano índico. Son unos islas verdes rodeadas de arena blanca"

c. "Quiero abirir un cafetría en china"

Por el contrario, se presentaron correcciones, como en (16a) y (16b) con los nombres no contables 'agua' e 'infusión', que son ítems léxicos que hacen referencia a entidades cotidianas, dentro de su nuevo dominio, el español. Hay un nombre contable 'flor' cuyo artículo fue corregido, también en términos de género:

(16)a. "esperando to el agua a hervisión"

b. “...esperando ela infusión a poco cliente"

c. "Aparece como un -una- flor"

\section{Conclusiones}

Si bien este un trabajo de caso, es interesante la manera en que estos estudiantes tratan de establecer las relaciones de concordancia entre los elementos de la frase nominal. Los problemas presentados se deben, principalmente, a que ambas lenguas involucradas, español y chino mandarín, pertenecen a tipologías diferentes, por lo que cada lengua 
tiene distintos elementos que conforman una frase nominal así como la manera en que las palabras están formadas, lo que les dificulta a los chino hablantes establecer las relaciones gramaticales entre los constituyentes de la frase nominal en español.

En general, la estructura de las frases nominales de los sino hablantes revelan que no tuvieron mayor problema en cuanto al orden de los elementos que constituyen estas frases, pues en general la formaron de la siguiente manera:

(17)FN: (ART) + (ADJ) + N + (ADJ) + (FP)

El problema como mencioné, radica en la ausencia de las relaciones de concordancia entre los mismos elementos que componen las frases nominales, sobre todo con respecto al género, debido a que, en la lengua materna, chino mandarín, no aparecen elementos, como los artículos, o los sufijos en español, que establezcan estas relaciones de género y número entre el nombre y los otros elementos.

Me parece que los resultados que se logren en un estudio a mayor escala serán de gran aportación tanto a maestros de español como alumnos que tengan interés de estudiarlo, pues ayudaría a proponer un método más adecuado de aprendizaje -más eficaz y rápido- para los alumnos, pues podrán conocer las particularidades de la lengua meta en comparación con las lenguas maternas.

\section{Referencias}

Alarcos, E. (1994). Gramática de la lengua española. Madrid: ESPASA.

Alcina, J. y Blecua, J. M. (2001). Gramática española. Barcelona: Editorial Ariel.

Bello, A. (1984). Gramática de la lengua castellana. Madrid: Editorial EDAF.

Charters, A. (2005). The Second Language Acquisition of Mandarin Nominal Syntax. Tesis de Doctorado en Lingüística, Universidad de Auckland.

Comrie, B. (1981). Universales del lenguaje y tipología lingüística: sintaxis y morfología. Madrid: Editorial Gredos.
Di Tullio, A. (2010) Manual de gramática de español. 3ra. Ed. Buenos Aires: Waldhuter Editores.

Gili, S. (1961). Curso superior de sintaxis española. Madrid: Biblograf.

González, C. y Herrero, M. C. (1997). Manual de gramática española. Gramática de la palabra, de la oración y del texto. Madrid: Editorial Castalia.

Gutiérrez, M. L. (2004). Problemas fundamentales de la gramática del español como 2/L. Madrid: Editorial Arco.

Li, Ch. N. y Thompson, S. A. (1989). Mandarin Chinese: A functional reference grammar. Berkeley: University of California Press.

Payne, T. E. (1997). Describing morphosyntax: a guide for field linguists. Cambridge: Cambridge University Press.

Po-Ching, Y. y Rimmington, D. (2004). Chinese: A comprehensive grammar. Londres y Nueva York: Routledge.

Real Academia Española. (2010). Nueva gramática de la lengua español. Madrid: Editorial Espasa-Calpe.

Yule, G. (2007). El lenguaje. Madrid: Ediciones Akal.

\section{Referencia citada}

Arcos, M. E. (2009). Análisis de errores, contrastivo e interlengua, en estudiantes brasileños de español como segunda lengua: verbos que rigen preposición y/o ausencia de ella. Tesis de doctorado.

Bartolomé, R. (2008). La adquisición y el aprendizaje de los modificadores nominales en el discurso escrito de estudiantes de español como L1 y L2. Revista Electrónica de Lingüística Aplicada, Número 7, 45-54.

Cadierno, T. (2010). El aprendizaje y la enseñanza de la gramática en el español como segunda lengua. MarcoELE, núm. 10, 1-18.

Liao, Y. y Fukuya, Y. J. (2004). La evitación de las frases verbales: el caso de los estudiantes chinos de inglés. Language Learning, 193-226.

Livinstone, K. A. y Ferreira, A. (2009). La efectividad de un modelo metodológico mixto para le enseñanzaaprendizaje de español como lengua extranjera. Boletín de Filología, Tomo XLIV, 89-118.

Madrigal, M. (2008). La escritura como proceso: metodología para la enseñanza de la expresión escrita en español como segunda lengua. Filología y Lingüística XXXIV, 127-141. 
Murillo, J. (2004). La pragmática y la enseñanza del español como segunda lengua. Educación, año/ vol. 28, núm. 002, 255-267.
Suárez, S. (2008). "Pedro comió la torta" vs. "Pedro se comió la torta": L2 adquisición de las construcciones del español télico "se". Anuario N 7, 227-295.

AUTOR

DANIELA GUTIÉRREZ BARRAZA Daniela Gutierrez Barraza, estudiante de Doctorado en Humanidades. Licenciatura en Lingüística y Máster en Humanidades en el área de estudios lingüísticos. Sus intereses investigativos han sido en estudios del español como lengua materna y, especialmente como segunda lengua. 